\title{
Globular Cluster Luminosity Functions and the Hubble Constant from WFPC2 Imaging: Galaxies in the Coma I Cloud ${ }^{1}$
}

\author{
Duncan A. Forbes \\ Lick Observatory, University of California, Santa Cruz, CA 95064 \\ and \\ School of Physics and Space Research, University of Birmingham, Edgbaston, Birmingham B15 2TT, United \\ Kingdom \\ Electronic mail: forbes@lick.ucsc.edu
}

\begin{abstract}
The membership of some galaxies in the nearby ( $\mathrm{d} \sim 12 \mathrm{Mpc}$ ) Coma I cloud is uncertain. Here we present globular cluster luminosity functions (GCLFs) from the Hubble Space Telescope for two bright ellipticals which may belong to this group. After fitting the GCLF, we find a turnover magnitude of $m_{V}^{0}=23.23 \pm 0.11$ for NGC 4278 and $m_{V}^{0}=23.07 \pm 0.13$ for NGC 4494. Our limiting magnitude is about two magnitudes fainter than these values, making this data among the most complete GCLFs published to date. The fitted GCLF dispersions $\left(\sim 1.1^{m}\right)$ are somewhat smaller than typical values for other ellipticals. Assuming an absolute turnover magnitude of $\mathrm{M}_{V}^{0}=-7.62$, and after applying a small metallicity correction, we derive distance modulii of $(\mathrm{m}-\mathrm{M})=30.61$ \pm 0.14 for NGC 4278 and $30.50 \pm 0.15$ for NGC 4494. These distance estimates are compared to other methods, and lie within the published range of values. We conclude that both galaxies lie at the same distance and are both members of the Coma I cloud.
\end{abstract}

\footnotetext{
${ }^{1}$ Based on observations with the NASA/ESA Hubble Space Telescope, obtained at the Space Telescope Science Institute, which is operated by AURA, Inc., under NASA contract NAS 5-26555
} 


\section{Introduction}

Although galaxies cover a wide range in properties, such as luminosity, Hubble type and environment, their globular cluster systems are remarkably similar in many respects. In particular, all globular cluster luminosity functions (GCLF) can be crudely represented by a Gaussian with a peak located at $\mathrm{M}_{V}$ $\sim-7.5$ and a width of $\sigma \sim 1.2$. The peak, or turnover magnitude, has been used with some success as an independent distance estimator to galaxies (see Jacoby et al. 1992). Its usefulness relies on the assumption that the turnover magnitude is universal for all galaxies. It has been suggested that the GCLF is not universal, but rather varies systematically with GC metallicity (Ashman, Conti \& Zepf 1995), the galaxy Hubble type (Secker \& Harris 1993) or galaxy environment (Blakeslee \& Tonry 1996). If any of these 'second parameter' effects are confirmed, then accurate distance estimates using the GCLF method will require an appropriate correction. In the case of GC metallicity, Ashman et al. (1995) have quantified the effects of metallicity on the turnover magnitude and show that once a metallicity correction $(\sim 0.1 \mathrm{mag})$ is applied, the GCLF gives distances in good agreement with other methods.

To fully resolve these issues, a number of high quality GCLFs are required covering a range of Hubble types and environments. One approach is to study galaxies that belong to a nearby group. This has the benefit of allowing the GCLF-determined distances to be directly compared with other distance methods for galaxies in the same group. Fleming et al. (1995) aimed to use this approach on the nearby Coma I cloud by examining the GCLF in NGC 4494 (E1) and NGC 4565 (Sb). Their results will be discussed below. The Coma I cloud is a small group of galaxies in the foreground of the well-known Coma cluster of galaxies (A1656). It is dominated by spiral galaxies but also includes a few ellipticals (Gregory \& Thompson 1977).

Here we examine the GCLFs of the two brightest ellipticals which may be members of the Coma I cloud, namely NGC 4278 and NGC 4494. Both galaxies contain a kinematically-distinct core which may have resulted from a merger. Using Hubble Space Telescope Wide Field and Planetary Camera 2 (WFPC2) data of Forbes et al. (1996), we can probe the GCLF in these galaxies to about 2 magnitudes beyond the turnover magnitude. The WFPC2 data have the ad- ditional advantages of very low background contamination with no blending of GCs in the central galaxy region. Our method is similar to that applied to the GCLF of NGC 4365 (Forbes 1996). In particular, we use the maximum likelihood code of Secker \& Harris (1993) to fit the GCLF and the absolute turnover magnitude determination of Sandage \& Tammann (1995), i.e. $\mathrm{M}_{V}^{0}=-7.62 \pm 0.08$. We compare the GCLF distance modulus with other methods and estimate the Hubble constant.

\section{Observations and Data Reduction}

Details of the WFPC2 data for the GCs in NGC 4278 and NGC 4494 are presented, along with 12 other ellipticals, in Forbes et al. (1996). From the $2 \times 500$ s F555W images, we use DAOPHOT (Stetson 1987) to detect GCs. Detection is based on fairly conservative limits of flux threshold, shape, sharpness and size. And after checking against a list of hot pixels, the final contamination from cosmic rays, foreground stars, background galaxies and hot pixels is less than a few percent. We did not apply any color selection to the GC lists. The GC magnitudes have been converted into Johnson $\mathrm{V}$ and corrected for Galactic extinction.

When examining the GCLF of NGC 4365 (Forbes 1996), we showed that the fraction of detected GCs with magnitude, once the threshold criterion is adjusted for a 0.3 mag sensitivity difference (Burrows et al. 1993), is similar for all four CCDs. For NGC 4494 we were able to use all CCDs, as the dust is confined to a small $\left(\sim 1^{\prime \prime}\right)$ ring which doesn't appear to affect any GCs (Carollo et al. 1996). In the case of NGC 4278, dust covers much of the PC CCD. We have therefore decided not to include GCs from the $\mathrm{PC}$ in this analysis.

\section{3. $\quad$ Modeling}

A full description of the modeling processes including completeness tests, photometric errors, background contamination and maximum likelihood fitting, are given in Forbes (1996). To summarize, we carried out simulations to test the ability of DAOPHOT to detect GCs on actual WFC images for both galaxies. These completeness tests, and the photometric error in measuring GC magnitudes, are given in Figure 1 for NGC 4278 and Figure 2 for NGC 4494. Both figures are similar to that found for NGC 4365 (Forbes 1996). The $50 \%$ completeness level is $\mathrm{V}=$ 
24.9 for NGC 4278 and $\mathrm{V}=24.8$ for NGC 4494. We ignore GCs with magnitudes fainter than these to avoid large incompleteness corrections. A small correction is made for background contamination based on similar exposure WFPC2 images from the Medium Deep Survey (Forbes et al. 1994). Finally we fit the background-corrected GCLF using the maximum likelihood code of Secker \& Harris (1993) which takes account of the completeness and photometric error variations with magnitude. We fit both Gaussian and $t_{5}$ distributions to the GCLF.

\section{Results and Discussion}

We have detected 241 GCs in NGC 4278 and 148 in NGC 4494 to the $50 \%$ completeness level. The results of fitting the GCLF of each galaxy, with the Gaussian and a $t_{5}$ functions, are summarized in Table 1 . In particular, the average turnover magnitudes are $\mathrm{m}_{V}^{0}=23.23 \pm 0.11$ for $\mathrm{NGC} 4278$ and $\mathrm{m}_{V}^{0}=$ $23.07 \pm 0.13$ for NGC 4494. As these magnitudes are statistically within the combined errors, this would indicate that both galaxies are at the same distance. The probability contours output from the maximum likelihood code for the Gaussian fit, over a range of 0.5-3 standard deviations, are shown in Figures 3 and 4. In Figures 5 and 6 we show the binned GCLF and our best-fit Gaussian superposed for each galaxy. Note that the fitting procedure does not use binned data but rather treats each data point individually. These figures clearly show that the $50 \%$ completeness limit is almost 2 magnitudes fainter than the turnover magnitude, giving us additional confidence that the turnover is well determined. This makes our data among the most complete, in terms of sampling the luminosity function, of GCLFs published to date. The number of 'missing' GCs, i.e. those fainter than the limiting magnitude, is $\leq 10 \%$.

Fleming et al. (1995) have recently investigated the GCLF of NGC 4494 and the Sb spiral NGC 4565. The aim of their study was to derive a GCLF distance for galaxies of different Hubble types located in the same group. This would allow a direct test of whether or not the GCLF turnover magnitude depends on Hubble type. They used the same CFHT $\sim 1^{\prime \prime} \mathrm{V}$ band images used by Simard \& Pritchet (1994) for a surface brightness fluctuation (SBF) study of these galaxies. The data consisted of two pointings for each galaxy. Using DAOPHOT for GC detection and completeness tests, their 50\% completeness limits for the two pointings were $\mathrm{V}=23.2,24.0$ for $\mathrm{NGC}$ 4494 and $\mathrm{V}=24.5,24.3$ for NGC 4565. For a Gaussian fit to their GCLF data of NGC 4494 they quote $m_{V}^{0}=23.6 \pm 0.4$ with $\sigma$ fixed at 1.4. The results for NGC 4565 are $m_{V}^{0}=22.63 \pm 0.2, \sigma=1.35 \pm 0.22$. In both cases there is considerable scatter in their faint magnitude bins (see their Figures 5 and 6). They concluded that NGC 4565 was in the Coma I cloud but that NGC 4494 lies in the background.

We now consider whether their data, and hence inferred distances, are consistent with our dataset. We first note that all Fleming et al. magnitudes should be $0.05^{m}$ brighter after applying a Galactic extinction correction. Starting with NGC 4494, for which their $50 \%$ completeness limit is comparable to their estimated turnover magnitude. For a Gaussian fit our data gives $m_{V}^{0}=23.05 \pm 0.13$ which is not consistent with their result. However, an important distinction is that they fixed $\sigma$ to be 1.4 (their data did not warrant fits to both $\sigma$ and $m_{V}^{0}$ ). It has been shown that uncertainties in $\sigma$ correspond to uncertainties in $m_{V}^{0}$, so that a change from $\sigma=1.4$ (Fleming et al. ) to 1.1 (us) would translate into a brighter $m_{V}^{0}$ by about $0.5^{m}$ (e.g. Hanes 1977; Secker \& Harris 1993). Thus the Fleming et al. GCLF would have a turnover of $m_{V}^{0}$ $\sim$ 23.05. An alternative, and perhaps more straight forward, way to show this is given by Figure 7 . This shows the Fleming et al. data for NGC 4494 and the best fit Gaussian to our data for NGC 4494. We include two Gaussians arbitrarily scaled up and down by a factor of two vertically (the turnover magnitude is held constant, but the dispersion is allowed to vary). This figure indicates that, within the error bars, the Fleming et al. data for NGC 4494 are consistent with a Gaussian that has a turnover of $m_{V}^{0}=23.05$. As a further test, we re-fit their data but excluded the faintest bin and allowed the Gaussian dispersion $\sigma$ to be a free parameter (along with the turnover magnitude and the normalization). This gave $\mathrm{m}_{V}^{0}=23.27$ \pm 0.4 (after extinction correction) and $\sigma=1.3 \pm 0.3$. We conclude that the ground-based dataset of Fleming et al. (1995) for NGC 4494 and our WFPC2 data are consistent, albeit within some large scatter.

Next we consider NGC 4565, in particular is it at the same distance as NGC 4494 (and NGC 4278)? Figure 7 shows the Fleming et al. data for the spiral NGC 4565. The data lie between the best fit Gaussian to our NGC 4494 data and one scaled down by a factor of two. Thus, as with NGC 4494, the groundbased data for NGC 4565 is consistent with a Gaus- 
sian of $m_{V}^{0}=23.05$. It is difficult to rule out the possibility that the turnover magnitude is brighter by $\sim 0.5^{m}$ (this difference is too large to be explained as metallicity effect Ashman et al. 1995).

We now calculate the distance modulus for our data from the apparent turnover magnitude. As noted in the introduction, Ashman et al. (1995) have advocated that a small correction be applied to the universal value based on GC metallicity. Such a metallicity correction has been shown by them to improve GCLF distance estimates. In the absence of spectroscopic measures, we can estimate the mean metallicity of a $\mathrm{GC}$ system assuming $[\mathrm{Fe} / \mathrm{H}]=5.051$ (V-I) - 6.096 (Couture et al. 1990). Using the mean color from Forbes et al. (1996), we derive $[\mathrm{Fe} / \mathrm{H}]=$ -0.79 for NGC 4278 and -0.84 for NGC 4494, giving metallicity corrections of $\Delta \mathrm{M}_{V}=0.16$ and 0.14 respectively. Thus we make the universal value of $\mathrm{M}_{V}^{0}$ $=-7.62 \pm 0.08$ (Sandage \& Tammann 1995) fainter by 0.16 or 0.14 magnitudes.

In Table 2 we summarize distance determinations from other workers for galaxies in the Coma I cloud. We include the GCLF distance for NGC 4565 from Fleming et al. (1995), after making a 0.05 Galactic extinction correction and assuming $\mathrm{M}_{V}^{0}=-7.62 \pm$ 0.08 with no metallicity correction. From V band surface brightness fluctuations, Simard \& Pritchet (1994) estimate $(\mathrm{m}-\mathrm{M})=30.88 \pm 0.3$ for NGC 4494, and $30.08 \pm 0.07$ for NGC 4565. Using the planetary nebulae luminosity function (PNLF) method, Jacoby, Ciardullo \& Harris (1996) get $30.54 \pm 0.05$ for NGC 4494 and $30.21 \pm 0.08$ for NGC 4565 . From the a mass model of the Virgo region and an assumption about the 'triple value ambiguity' in velocity, Tully \& Shaya (1984) give distances to NGC 4494 and NGC 4565 which correspond to 30.34 and 30.21 respectively. We will assume an error of $\pm 0.3^{m}$ on these estimates. Finally, Aaronson \& Mould (1983) find $(\mathrm{m}-\mathrm{M})=30.42$ \pm 0.32 for several spiral galaxies in the direction of the Coma I cloud using the infrared Tully-Fisher relation.

The distance modulus for NGC 4494 ranges from $(\mathrm{m}-\mathrm{M})=30.34$ (mass models of Tully \& Shaya 1984) to 30.88 (SBF work of Simard \& Pritchet 1994) with a weighted mean of $30.54 \pm 0.07$. Our value for NGC $4278(30.61 \pm 0.14)$ is consistent with the NGC 4494 mean. Most recently, I band SBF measurements also indicate that NGC 4278 and NGC 4494 have essentially the same distance modulus, albeit at the upper range of values listed in Table 2 (Tonry 1996). For
NGC 4565 the weighted mean distance modulus is $30.10 \pm 0.05$. This is $0.4^{m}$ or $2 \mathrm{Mpc}$ closer than NGC 4494. This suggests that NGC 4565 lies in the foreground relative to NGC 4494.

It has been suggested that the Coma I cloud may consist of two sub-groups, one around NGC 4565 and the other associated with the S0 galaxy NGC 4274 and NGC 4278 (de Vaucouleurs 1976). This claim was questioned by Gregory \& Thompson (1977) who found no particular evidence that the Coma I cloud formed two sub-groups. However, they did note that the group formed a bar-like structure of dimensions $0.9 \times 2.5 \mathrm{Mpc}$ and that the galaxies with the lower velocities tend to be systematically fainter, i.e. located further from us. Table 2 shows that in each case, other workers found NGC 4494 to be more distant than NGC 4565 for a given distance method. Our preferred interpretation is that both NGC 4494 and NGC 4278 are at the same distance and are located at the far end of the Coma I cloud, whereas NGC 4565 is located $2 \mathrm{Mpc}$ closer at the front end of the group.

Aaronson \& Mould (1983) caution that the redshifts for galaxies in the Coma I cloud may not correlate well with distance, given the proximity of the group to the Virgo cluster. Nevertheless, we will attempt to estimate the Hubble constant from our measurements. If we calculate the mean velocity of the eight galaxies associated with NGC 4274 sub-group (de Vaucouleurs 1976), and include NGC 4494, we get $880 \mathrm{~km} \mathrm{~s}^{-1}$. The correction for motion with respect to the Local Group using solution number 2 of Yahil, Tammann \& Sandage (1977) is $\sim-40 \mathrm{~km} \mathrm{~s}^{-1}$, giving $840 \mathrm{~km} \mathrm{~s}^{-1}$. The Virgocentric infall component from Tammann \& Sandage (1985) is $\sim 200 \mathrm{~km} \mathrm{~s}^{-1}$, which gives a corrected recession velocity of $1020 \mathrm{~km} \mathrm{~s}^{-1}$. Using a distance modulus of 30.54 and this corrected velocity we estimate a Hubble constant of $\sim 80 \mathrm{~km}$ $\mathrm{s}^{-1} \mathrm{Mpc}^{-1}$.

Finally, we have derived the local (within 100 " radius) and total GC specific frequency $(S)$ for each galaxy following the method described in Forbes (1996). These give similar results of $\sim 5$ for NGC 4278 and $\sim 2$ for NGC 4494. The richness of the GC system around NGC 4494 appears to lower than that of a typical elliptical ( $S \sim 5$; van den Bergh 1995).

\section{Conclusions}

Using WFPC2 data of Forbes et al. (1996) we have fit the globular cluster luminosity function (GCLF) of 
two ellipticals, NGC 4278 and NGC 4494. The first of which is generally thought to lie in the Coma I cloud, whereas the latter has been suggested to lie in the background. Both the Gaussian and $t_{5}$ profile fits give similar results, namely a turnover magnitude of $\mathrm{m}_{V}^{0}=23.23 \pm 0.11$ for NGC 4278 and $\mathrm{m}_{V}^{0}$ $=23.07 \pm 0.13$ for NGC 4494. The fitted dispersions $\left(\sigma \sim 1.1^{m}\right)$ are somewhat smaller than typical values for other ellipticals. The limiting magnitude, as determined by completeness tests, is about 2 magnitudes fainter than these values. We derive distance modulii of $30.61 \pm 0.14$ and $30.50 \pm 0.15$ for NGC 4278 and NGC 4494 respectively, assuming an absolute turnover magnitude of $\mathrm{M}_{V}=-7.62 \pm 0.08$ from Sandage \& Tammann (1995) and a small metallicity correction based on the precepts of Ashman et al. (1995). We compare our distance measure with the ground-based GCLF study of Fleming et al. (1995) and other distance determinations for galaxies in the Coma I cloud. Our distance modulii lie within the range of published values. We conclude that both NGC 4278 and NGC 4494 are members of the Coma I cloud, and speculate that they lie at the far end of a bar structure; the near end of which is associated with NGC 4565. Finally, we make a rough estimate of the Hubble constant and globular cluster specific frequency from our data.

\section{Acknowledgments}

We are particularly grateful to J. Secker for the use of his maximum likelihood code and useful suggestions. We also thank J. Blakeslee, J. Brodie and C. Grillmair for helpful discussions. The referee is thanked for several suggestions that have improved the paper. This research was funded by the HST grant AR-05794.01$94 \mathrm{~A}$

\section{References}

Aaronson, M., \& Mould, J. 1983, ApJ, 265, 1

Ashman, K. M., Conti, A., \& Zepf, S. E. 1995, AJ, 110, 1164

Blakeslee, J. P., \& Tonry, J. L. 1996, ApJ, in press

Burrows, C., et al. 1993, Hubble Space Telescope Wide Field and Planetary Camera 2 Instrument Handbook, STScI

Carollo, C. M., Franx, M., Illingworth, G. D., \& Forbes, D. A. 1996, ApJ, submitted

Couture, J., Harris, W. E., \& Allwright, J. W. B., 1990, ApJS, 73, 671

de Vaucouleurs, G. 1976, Stars and Stellar Systems, edited by A. Sandage, M. Sandage and J. Kristian
(Chicago: University of Chicago Press) v9, p557

Forbes, D. A. 1996, AJ, in press

Forbes, D. A., Elson, R. A. W., Phillips, A. C., Illingworth, G. D. \& Koo, D. C. 1994, ApJ, 437, L17

Forbes, D. A., Franx, M., Illingworth, G. D., \& Carollo, C. M. 1996, ApJ, in press

Fleming, D. E. B., Harris, W. E., Pritchet, C. J., \& Hanes, D. A. 1995, AJ, 109, 1044

Gregory, S. A., \& Thompson, L. A. 1977, ApJ, 213, 345

Hanes, D. A. 1977, MNRAS, 180, 309

Jacoby, G. H., et al. 1992, PASP, 104, 599

Jacoby, G. H., Ciardullo, R., \& Harris, W. E. 1996, ApJ, 462, 1

Sandage, A., \& Tammann, G. A. 1995, ApJ, 446, 1

Secker, J., \& Harris, W. E. 1993, AJ, 105, 1358

Simard, L., \& Pritchet, C. J. 1994, AJ, 107, 503

Stetson, P. B., 1987, PASP, 99, 191

Tammann, G. A., \& Sandage, A. 1985, ApJ, 294, 81

Tonry, J. L. 1996, The Extragalactic Distance Scale workshop held at Space Telescope Science Institute

Tully, R. B., \& Shaya, E. J. 1984, ApJ, 281, 31

van den Bergh, S. 1995, AJ, 110, 2700

Yahil, A., Tammann, G. A., \& Sandage, A. 1977, ApJ, 217, 903 

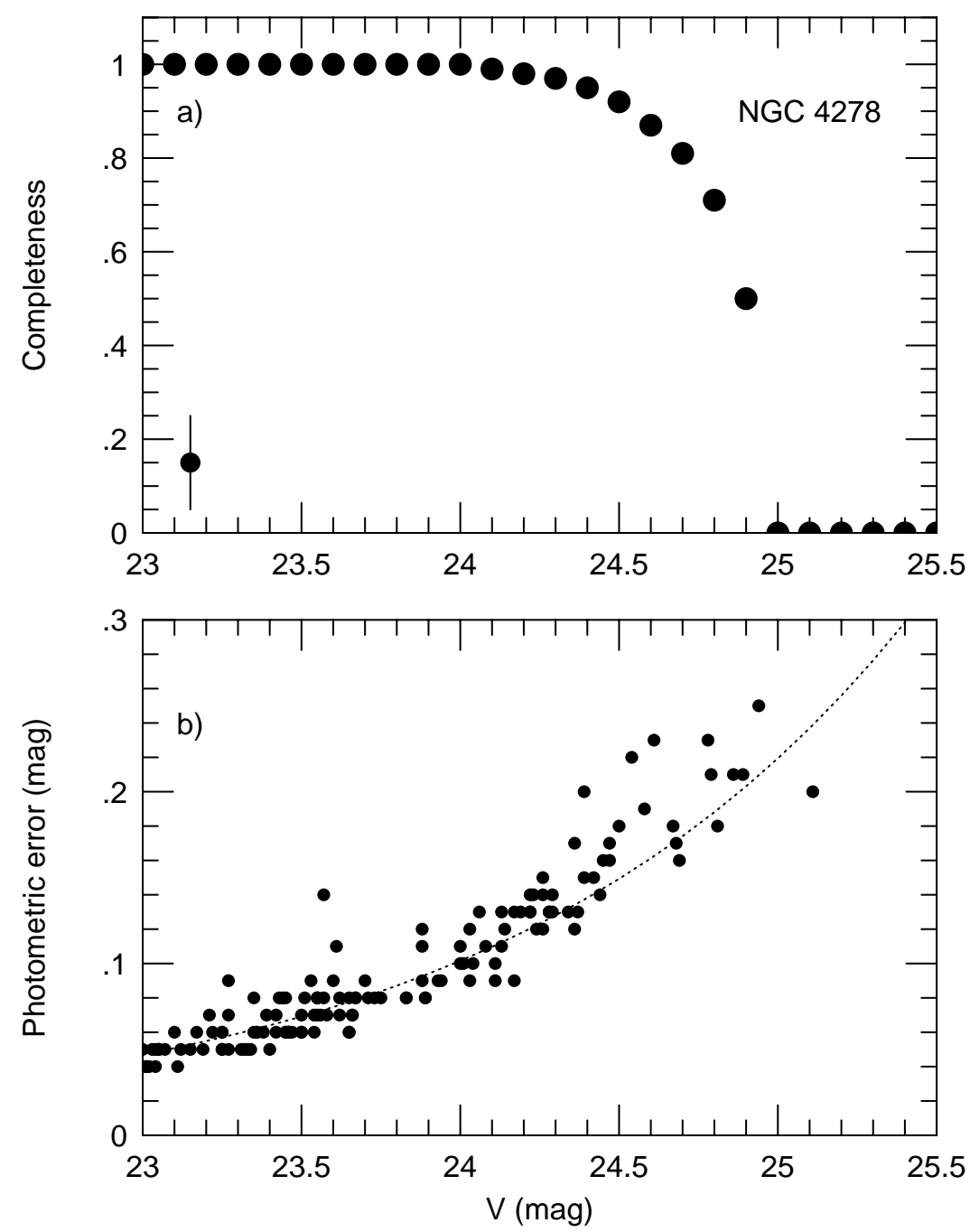

Fig. 1.- Completeness function for NGC 4278 from simulations. Circles show the fraction of simulated GCs detected in 0.1 magnitude bins. A typical error bar is shown in the lower left. b) Photometric error for NGC 4278 as a function of GC V magnitude determined from DAOPHOT. Circles show the data points, and the dashed line an exponential fit to the data of the form p.e. $=\exp [\mathrm{a}(\mathrm{V}-\mathrm{b})]$. 

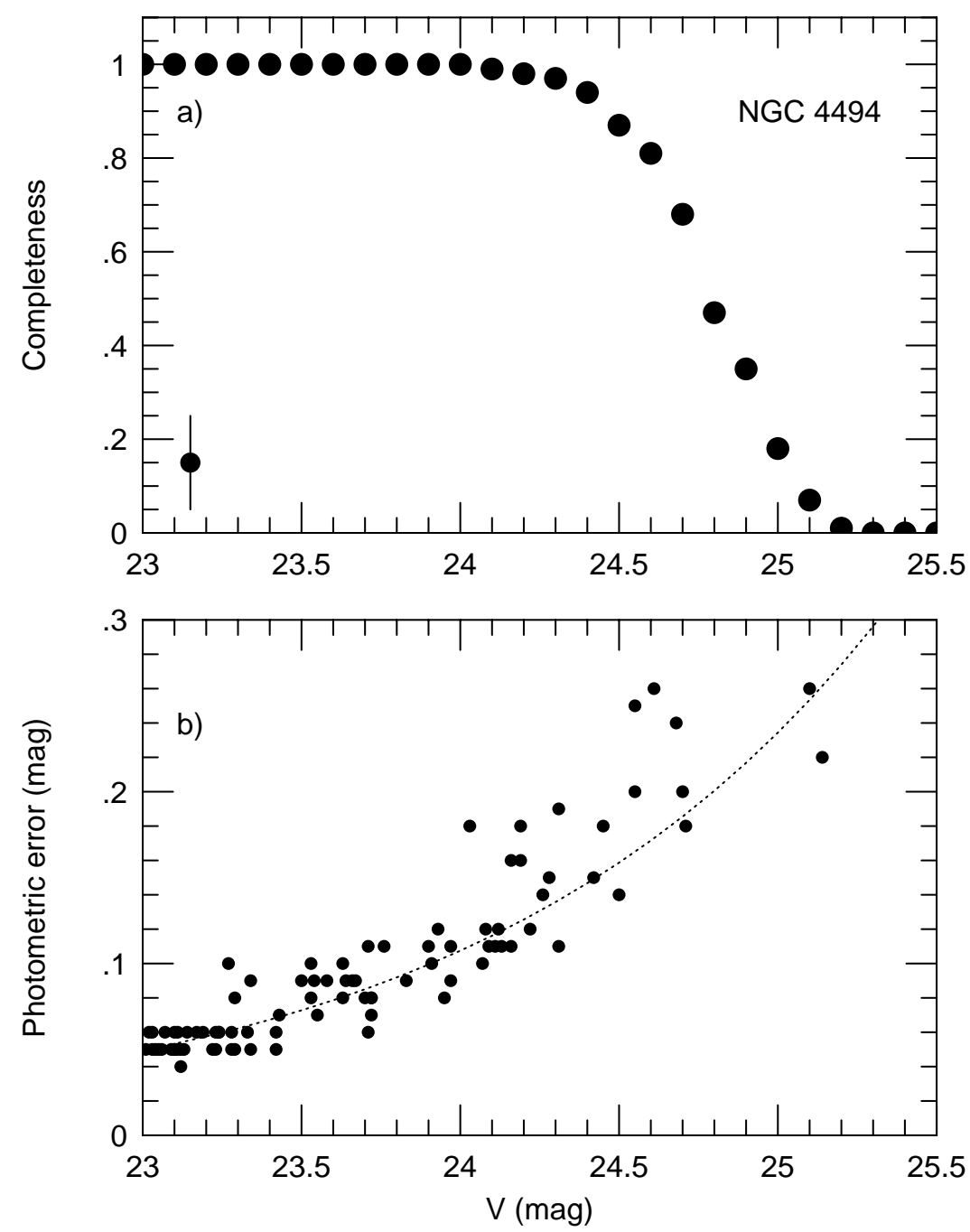

Fig. 2.- Completeness function for NGC 4494 from simulations. Circles show the fraction of simulated GCs detected in 0.1 magnitude bins. A typical error bar is shown in the lower left. b) Photometric error for NGC 4494 as a function of GC V magnitude determined from DAOPHOT. Circles show the data points, and the dashed line an exponential fit to the data of the form p.e. $=\exp [\mathrm{a}(\mathrm{V}-\mathrm{b})]$. 


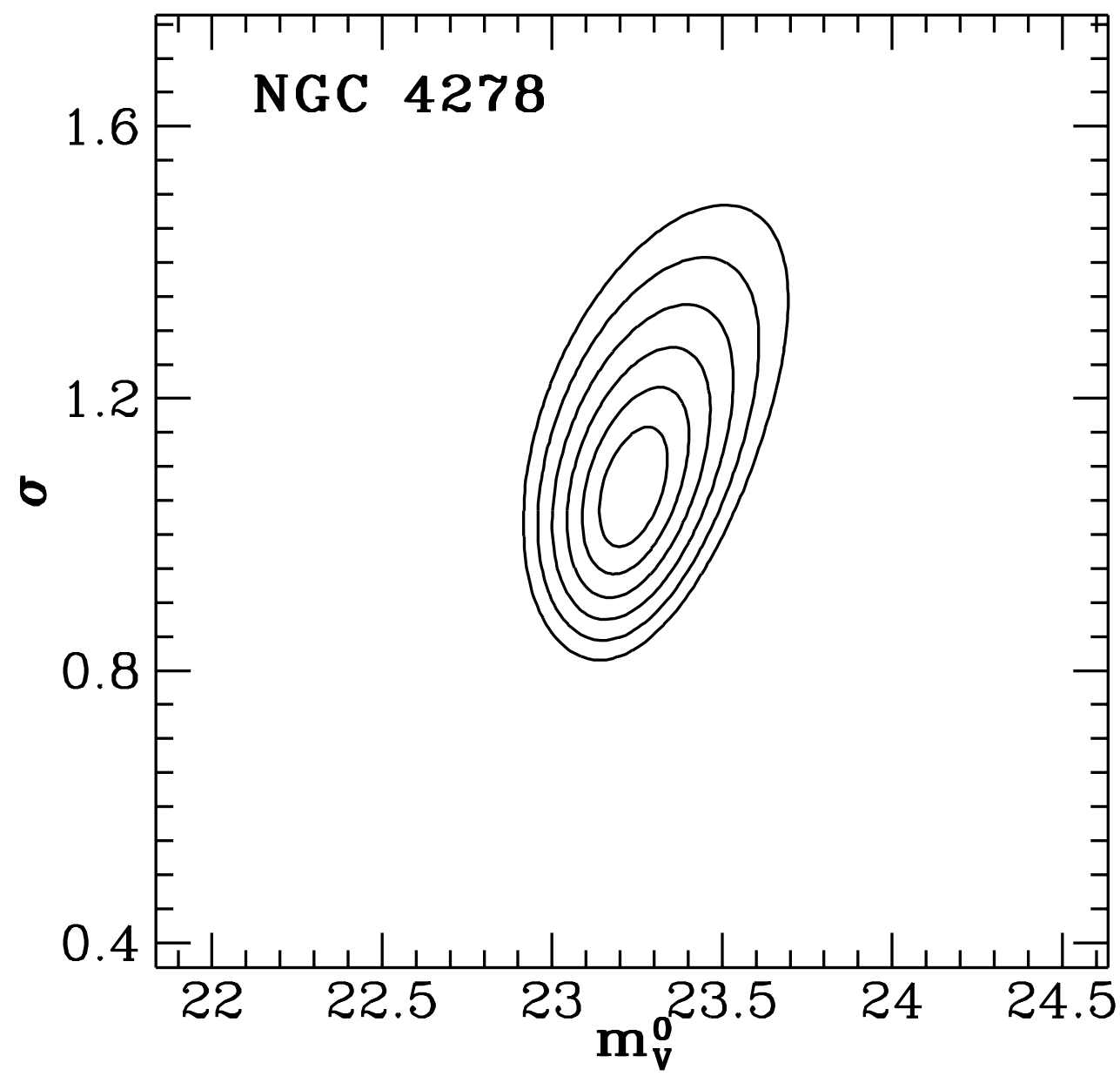

Fig. 3.- Probability contours for a Gaussian fit to the globular cluster luminosity function of NGC 4278. Contours represent 0.5 to 3 standard deviations probability limits from the best estimate (see Table 1). 


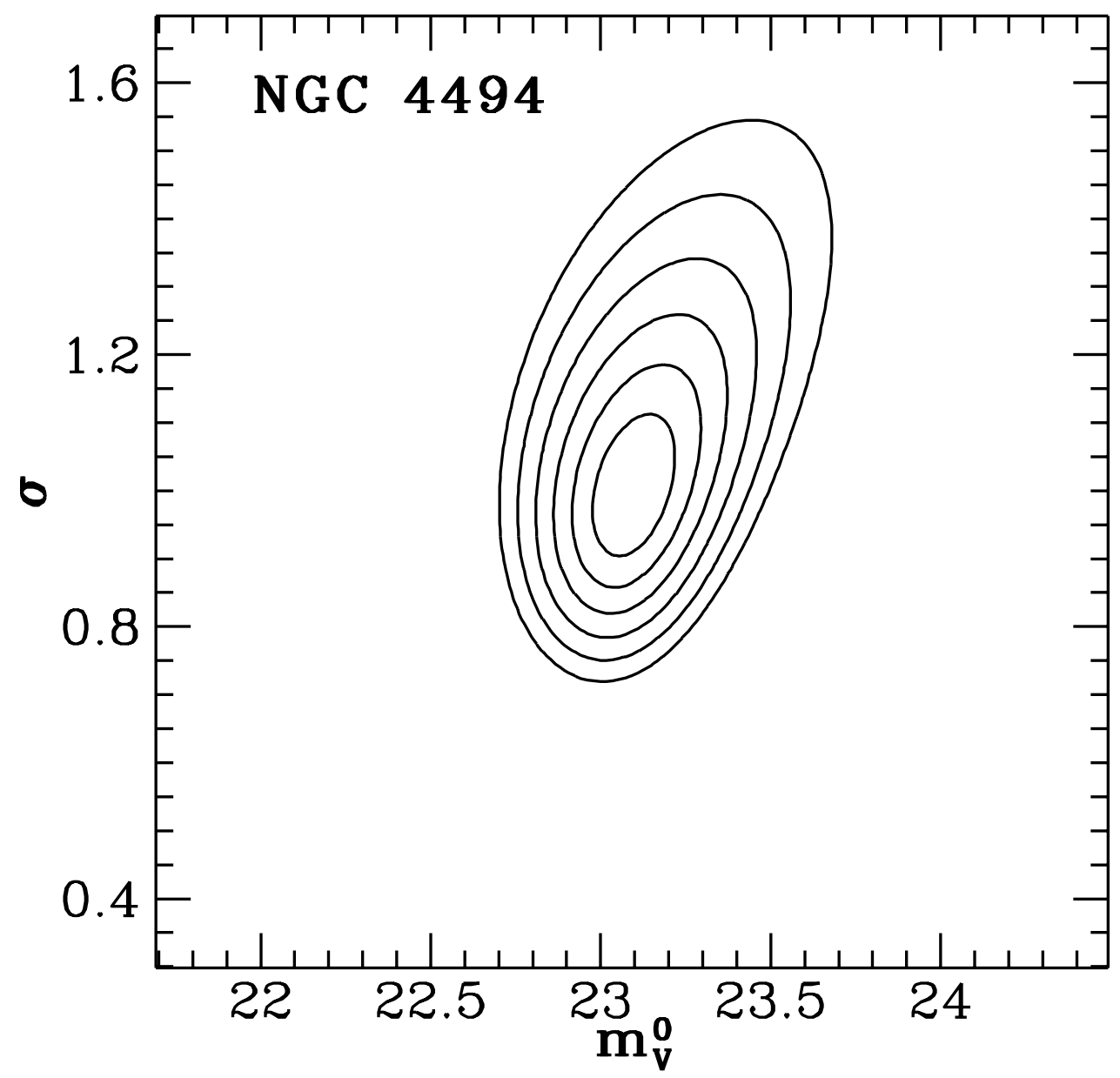

Fig. 4.- Probability contours for a Gaussian fit to the globular cluster luminosity function of NGC 4494. Contours represent 0.5 to 3 standard deviations probability limits from the best estimate (see Table 1). 


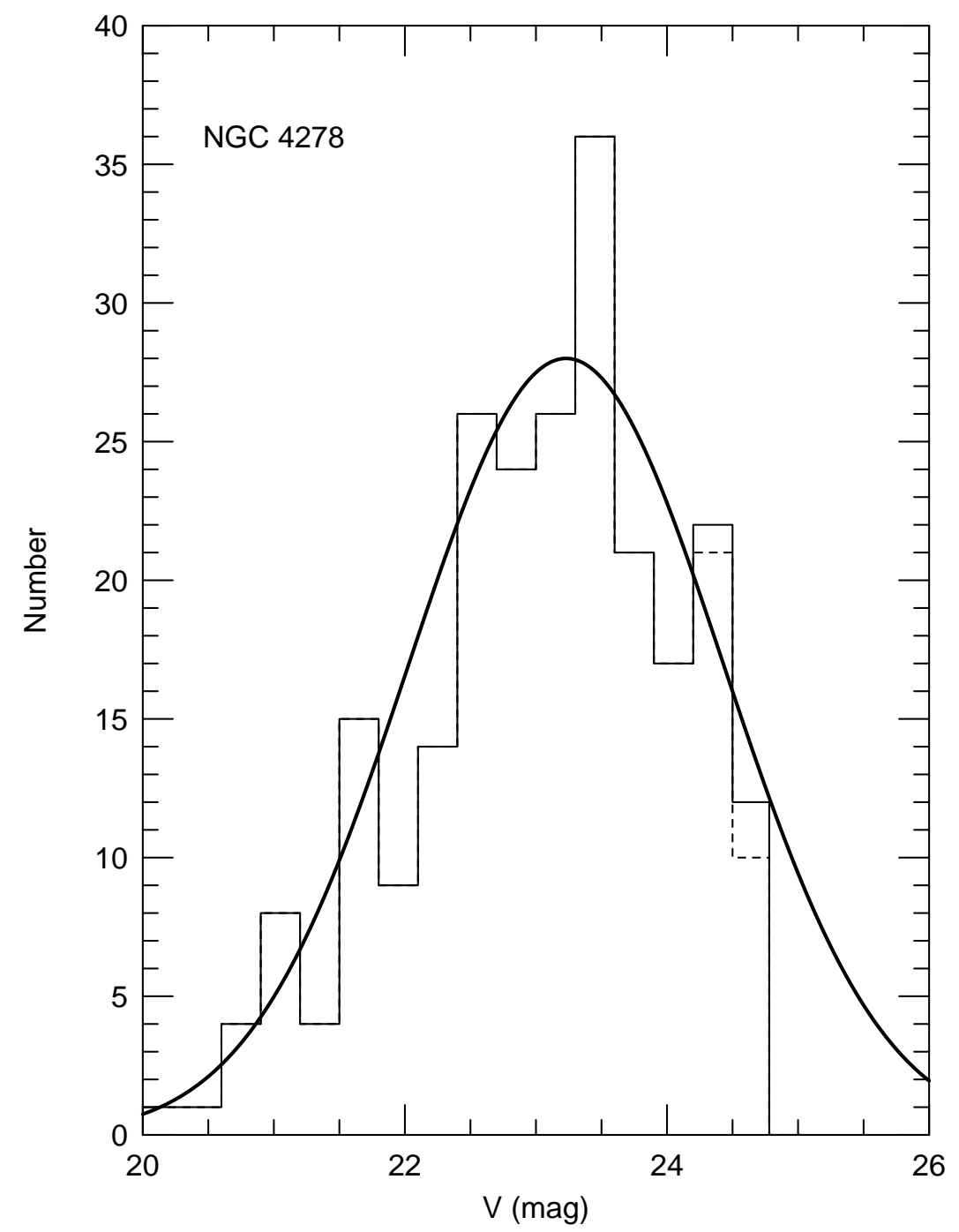

Fig. 5.- Globular cluster luminosity function for NGC 4278. The raw data is shown by a dashed line, and by a thin solid line after completeness correction has been applied. The maximum likelihood best fit Gaussian profile, which includes the effects of photometric error and background contamination, is superposed as a thick solid line. Note that the fitting procedure does not use binned data. 


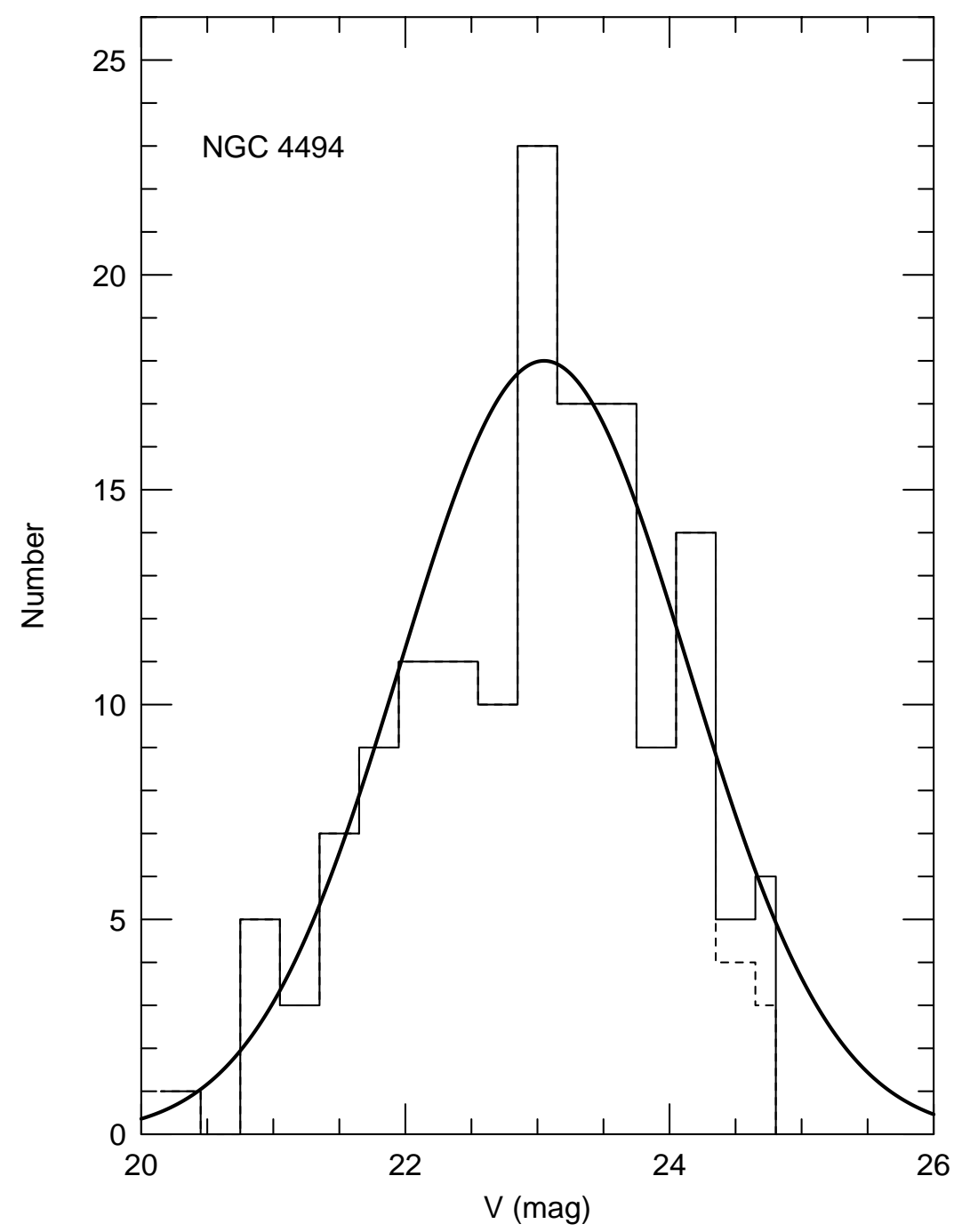

Fig. 6.- Globular cluster luminosity function for NGC 4494. The raw data is shown by a dashed line, and by a thin solid line after completeness correction has been applied. The maximum likelihood best fit Gaussian profile, which includes the effects of photometric error and background contamination, is superposed as a thick solid line. Note that the fitting procedure does not use binned data. 


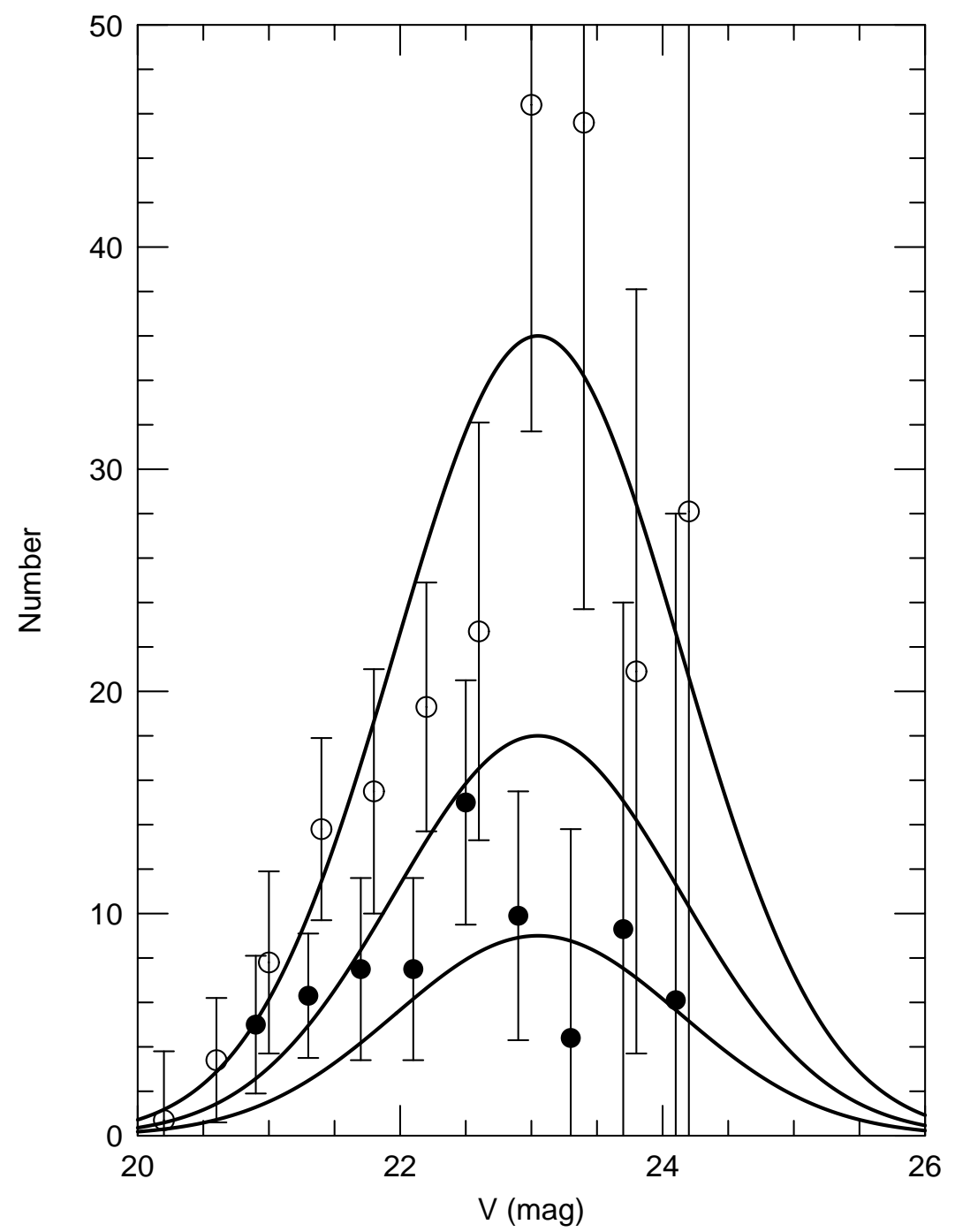

Fig. 7.- Comparison of the ground-based data from Fleming et al. (1995) with our data for NGC 4494. Open circles represent the Fleming et al. data for NGC 4494, and the filled circles for NGC 4565. The three solid lines represent our best fit Gaussian to the WFPC2 data on NGC 4494, and scaled up and down arbitrarily by a factor of two (the turnover magnitude is held fixed but the dispersion is allowed to vary). Within the large scatter, the ground-based data for NGC 4494 and NGC 4565 could be consistent with a turnover magnitude of $m_{V}^{0}=23.05$, as found from the WFPC2 data. 
TABLE 1. Globular Cluster Luminosity Function Parameters

\begin{tabular}{|c|c|c|c|c|}
\hline $\begin{array}{c}\text { Galaxy } \\
\text { (1) }\end{array}$ & $\begin{array}{c}m_{G}^{0} \\
(\mathrm{mag}) \\
(2)\end{array}$ & $\underset{(\mathrm{mag})}{\sigma_{G}}$ & $\begin{array}{c}m_{t}^{0} \\
(\mathrm{mag}) \\
(4)\end{array}$ & $\underset{(5)}{\sigma_{t}}$ \\
\hline NGC 4278 & $23.22 \pm 0.11$ & $1.21 \pm 0.09$ & $23.23 \pm 0.11$ & $1.07 \pm 0.09$ \\
\hline NGC 4494 & $23.05 \pm 0.13$ & $1.09 \pm 0.11$ & $23.09 \pm 0.13$ & $1.01 \pm 0.11$ \\
\hline
\end{tabular}

Notes to Table 1.

(1) Galaxy name; (2) Apparent magnitude of the GCLF turnover from a Gaussian fit; (3) Dispersion of the GCLF from a Gaussian fit; (4) Apparent magnitude of the GCLF turnover from a $t_{5}$ fit; (5) Dispersion of the GCLF from a $t_{5}$ fit.

TaBle 2. Distance Determinations for Galaxies in the Coma I Cloud

\begin{tabular}{cccc}
\hline \hline Galaxy & $\begin{array}{c}(\mathrm{m}-\mathrm{M}) \\
(\mathrm{mag}) \\
(1)\end{array}$ & $\begin{array}{c}(\text { Method }) \\
(3)\end{array}$ & $\begin{array}{c}\text { Ref. } \\
(4)\end{array}$ \\
\hline NGC 4278 & $30.61 \pm 0.14$ & GCLF & This work \\
NGC 4494 & $30.50 \pm 0.15$ & GCLF & This work \\
NGC 4494 & $30.88 \pm 0.3$ & SBF & Simard \\
NGC 4494 & $30.54 \pm 0.05$ & PNLF & Jacoby \\
NGC 4494 & 30.34 & Mass model & Tully \\
NGC 4565 & $30.20 \pm 0.22$ & GCLF & Fleming \\
NGC 4565 & $30.08 \pm 0.07$ & SBF & Simard \\
NGC 4565 & $30.12 \pm 0.10$ & PNLF & Jacoby \\
NGC 4565 & 30.21 & Mass model & Tully \\
Coma Spirals & $30.42 \pm 0.32$ & IRTF & Aaronson \\
& & & \\
\hline \hline
\end{tabular}

Notes to Table 2.

(1) Galaxy name; (2) Distance modulus (see text for details); (3) Distance modulus method, GCLF = globular cluster luminosity function, SBF = surface brightness functions, PNLF = planetary nebulae luminosity fucntion, IRTF = infrared Tully-Fisher; (4) Reference (Fleming et al. 1995, Simard \& Pritchet 1994, Jacoby et al. 1996, Tully \& Shaya 1984). 